\section{Coming Events}

2011

Microscopy Conference MC 2011

August 28-September 11, 2011

Kiel, Germany

www.mc2011.de

\section{Multinational Congress on Microscopy}

September 4-9, 2011

Urbino, Italy

www.mcm2011urbino.it

\section{ICXOM21}

September 5-8, 2011

Campinas, Brazil

icxom21.lnls.br

\section{EMAG 2011}

September 6-9, 2011

Birmingham, UK

www.emag-iop.org

National Society for Histotechnology September 16-21, 2011

Cincinnati, $\mathrm{OH}$

www.nsh.org

\section{FEMMS 2011}

September 18-23, 2011

Sonoma County, CA

www.femms2011.llnl.gov

\section{CIASEM 2011}

September 25-30, 2011

Mérida, Mexico

www.ciasem.com

\section{Neuroscience 2011}

November 12-16, 2011

Washington, DC

www.sfn.org

\section{MRS Fall Meeting 2011}

November 26-October 2, 2011

Boston, MA

www.mrs.org

American Society for Cell Biology December 3-7, 2011

Denver Convention Center, CO

www.ascb.org/meetings

\section{2}

Microscopy \& Microanalysis 2012

July 29-August 2, 2012

Phoenix, AZ

\section{3}

Microscopy \& Microanalysis 2013

August 4-8, 2013

Indianapolis, IN

2014

Microscopy \& Microanalysis 2014

August 3-7, 2014

Hartford, CT

\section{More Meetings and Courses}

Check the complete calendar near the back of this magazine and in the MSA journal Microscopy and Microanalysis.

\title{
Better Protein Localization
}

\author{
Stephen W. Carmichael ${ }^{1 *}$ and Philip Oshel ${ }^{2}$ \\ ${ }^{1}$ Mayo Clinic, Rochester, MN 55905 \\ ${ }^{2}$ Central Michigan University, Mt. Pleasant, MI 48859 \\ * carmichael.stephen@mayo.edu
}

Localizing specific proteins within cells, tissues, and organisms has been a goal of microscopists for generations. In the early 1990s, a breakthrough was made when a molecule originally derived from a jellyfish was introduced as a probe for fluorescence microscopy. This molecule is green fluorescent protein (GFP), and it has become well known for its usefulness in localizing proteins at the level of the light microscope. It is also well known that electron microscopy (EM) offers far superior spatial resolution over light microscopy, but the application of probes to localize specific proteins has required antibodies conjugated with colloidal metals (such as gold). Delivery of antibodies into the cell commonly requires detergents to permeabilize the cell membrane, which compromises the ultrastructural detail. Another breakthrough was recently published on-line by Xiaokun Shu, Varda Lev-Ram, Thomas Deerinck, Yingchuan Qi, Ericka Ramko, Michael Davidson, Yishi Jin, Mark Ellisman, and Roger Tsien [1]: they have developed a method similar to using GFP for light microscopy, but for specifically tagging proteins at the EM level.

The essential molecule for this technique is miniSOG (mini singlet oxygen generator); this is a small (106 amino acids), genetically encodable protein that does not need exogenous cofactors to fluoresce and generate ${ }^{1} \mathrm{O}_{2}$ (singlet oxygen) when exposed to blue light. Shu et al. began with the concept that the domain (LOV, for light, oxygen, and voltage) of phototropin that binds flavin mononucleotide could be converted into a molecule that generates ${ }^{1} \mathrm{O}_{2}$. This was accomplished by mutagenesis of the phototropin LOV domain in Arabidopsis thaliana. They then manipulated the resulting molecule by shuffling the DNA to produce miniSOG, which resulted in an increased fluorescent brightness. This miniSOG was then fused with the target protein, which allows for efficient labeling that can be visualized by fluorescent microscopy, in the same manner as GFP-target fusion proteins. However, the real advantage is that the illumination of miniSOG generates sufficient ${ }^{1} \mathrm{O}_{2}$ to locally catalyze the polymerization of diaminobenzidine (DAB) into an osmiophilic reaction product that can be visualized by EM. Furthermore, this can be done on specimens fixed by conventional techniques that yield images of detailed ultrastructure.

Shu et al. demonstrated correct localization of well-understood proteins ( $\alpha$-actinin, histone $2 \mathrm{~B}$, part of cytochrome $c$, and connexin 43 [Figures 1A and 1B]) tagged with miniSOG in cultured cells. They also localized cytochrome $c$ in a multicellular organism
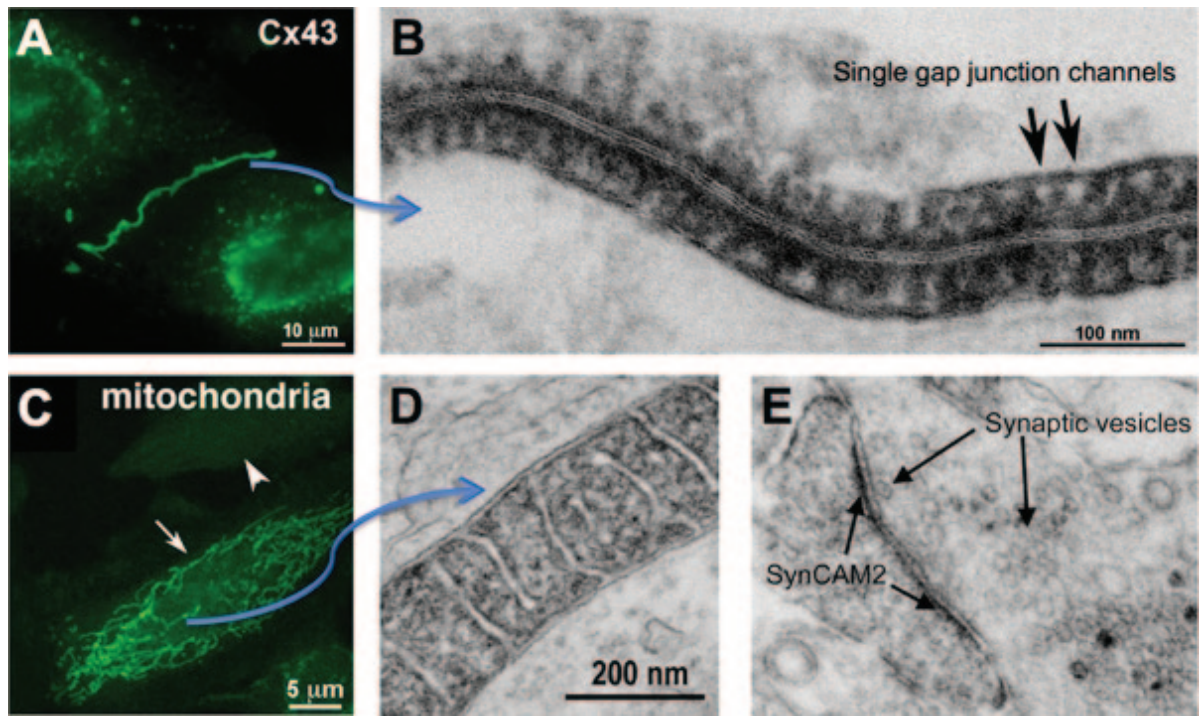

Figure 1: $A$ and $B$ show light microscopic and EM localization, respectively, of Cx43, and C and D show mitochondrial-targeted miniSOG. E shows EM localization of SynCAM2. 


\section{Revealing Interfaces}

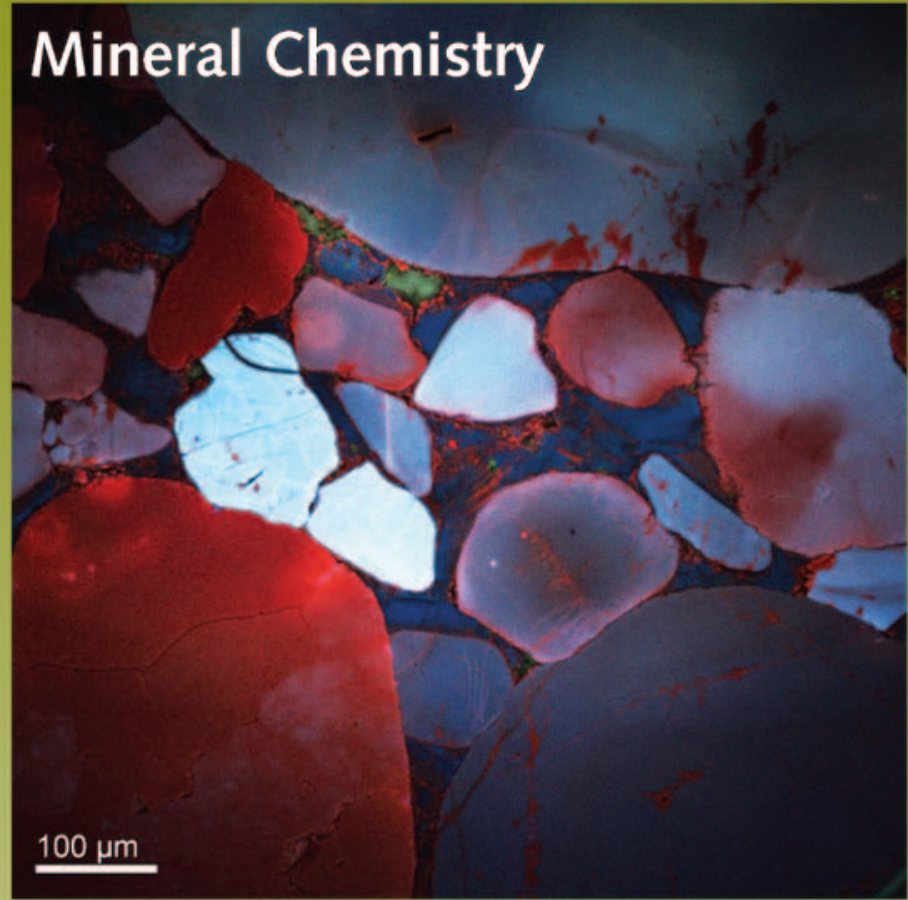

\section{MEMS}

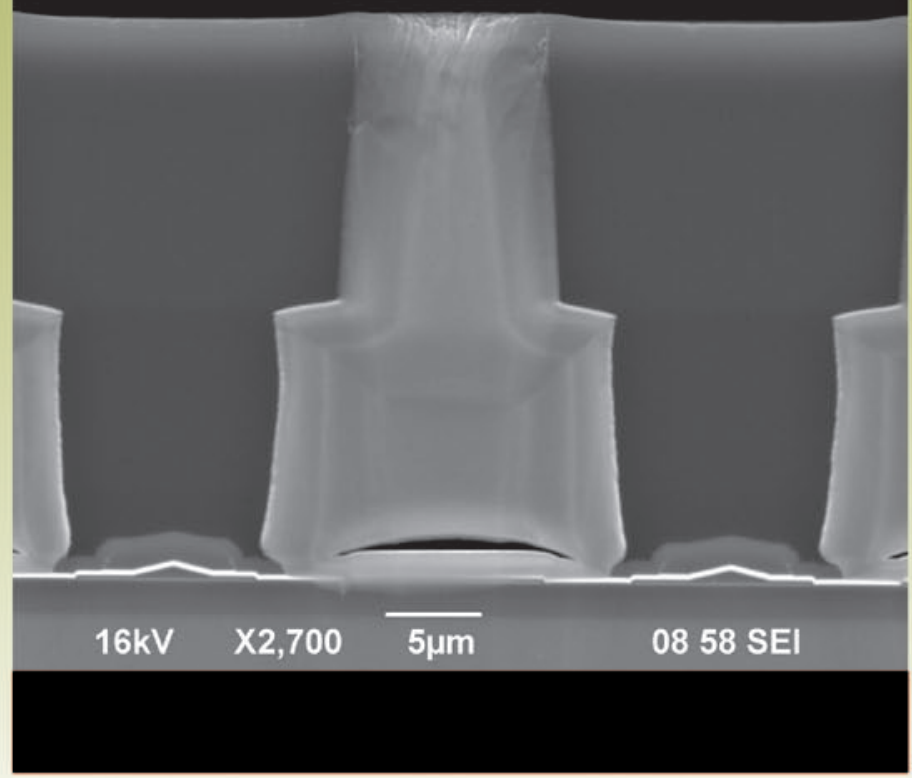

Mineral Chemistry: Quartz arenite polished section cathodoluminescence image prepared using the Gatan Ilion*'"' and imaged with Gatan ChromaCL'" imaging system. Image courtesy of Dr. J. Schieber, Indiana University. Electronic Materials: Active region of a commercial LED prepared using the Gatan Ilion ${ }^{+{ }^{+1}}$. Grain Orientation: SEM cross section of copper indexed to $98 \%$ with EBSD and prepared using the Gatan llion ${ }^{+7}$. MEMS: SEM cross section of an ink jet printer head prepared using the Gatan llion ${ }^{\text {tw" }}$.

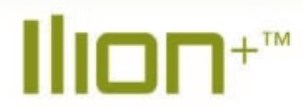

Surface Preparation for SEM Cross Section and Planar Viewing

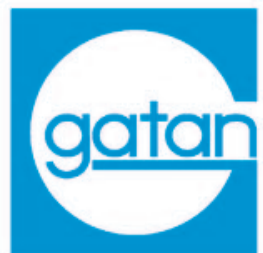

ANALYTICAL TEM

DIGITAL IMAGINC

SPECIMEN PREPARATION

TEM SPECIMEN HOLDERS

SEM PRODUCTS

SOFTWARE

www.gatan.com
Electronic Materials

\section{Grain Orientation}


(C. elegans) (Figure 1C and 1D). Then they localized the cell-adhesion molecules SynCAM1 and SynCAM2. They then used the miniSOG with the relatively new technique of "serial block-face scanning electron microscopy" to reveal the three-dimensional distribution of the SynCAMs in prenatal mouse brains (Figure 1E). In short, they demonstrated that SynCAM1 and SynCAM2 are localized to pre- and post-synaptic membranes, respectively. This technique shows great promise for establishing the 3-D architecture of diverse molecules in neuronal synapses, which will indeed be a complex task! Further, if the $\mathrm{DAB}$ and $\mathrm{OsO}_{4}$ do not quench the fluorescence of miniSOG, the target protein could possibly be imaged using a cathodoluminescence detector in the EM, as well as by simultaneous EM imaging. This could provide more evidence that the electron-dense DAB product is truly co-located with the miniSOG molecule, and hence the target protein.

At the end of their abstract, Shu et al. concluded that "MiniSOG may do for EM what Green Fluorescent Protein did for fluorescence microscopy." Considering the fact that the senior author, Dr. Tsien, shared the Nobel Prize in Chemistry in 2008 for his role in the discovery and development of GFP and other fluorescent tags, their statement is no idle boast [2].

\section{References}

[1] X Shu, V Lev-Ram, TJ Deerinck, Y Qi, EB Ramko, MW Davidson, Y Jin, MH Ellisman, and RY Tsien, PLoS Biol 9(4), e1001041. DOI: 10:1371/journal.pbio.1001041

[2] The authors gratefully acknowledge Dr. Xiaokun Shu for reviewing this article.
Minus $K^{\diamond}$ Technology's Negative-Stiffness vibration isolators have been selected for ground testing of the James Webb Space Telescope (JWST).

\section{Why have over 2,000 scientists \\ in 35 countries selected
Minus $K^{\circledR}$ vibration isolators? \\ in 35 countries selected
Minus $K^{\circledR}$ vibration isolators?}

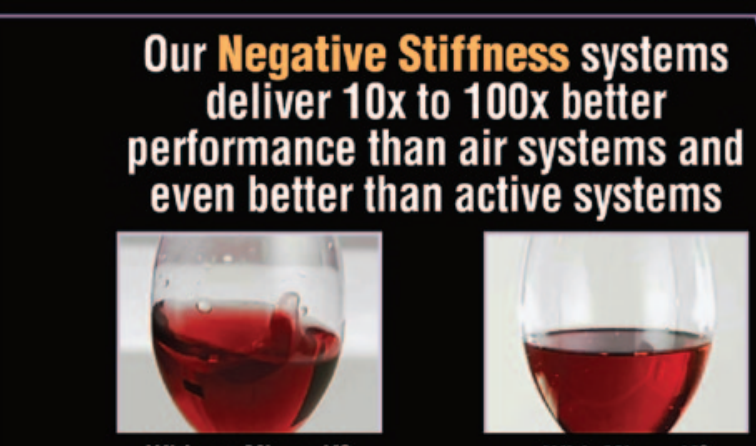

Without Minus $\mathrm{K}^{\odot}$

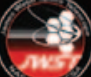

(1)

\section{Our Negative Stiffness systems deliver $10 x$ to $100 x$ better \\ performance than air systems and even better than active systems}

The best performance and the lowest price. That's hard to beat!

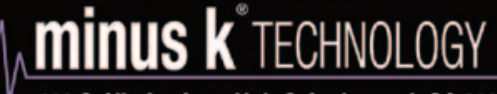

460 S. Hindry Ave., Unit C, Inglewood, CA 90301

Tel: 310-348-9656 Fax: 310-348-9638

sales@minusk.com · www.minusk.com

\section{FIVE GENERATIONS OF PERFORMANCE...REFINED}

\section{The new Desk V HP sputtering tool.}

With an enhanced sputter head, larger mechanical pump, a more powerful PLC and a larger power supply, the all new Desk V HP is generations ahead of the competition.

Features:

- Short deposition times

- Consistent deposition parameters

- Enhanced touchscreen controls

- Film thickness control

- Etch mode for sample cleaning

- Wide variety of coating materials

- Compact benchtop design

- 85 LPM Mechanical Pump

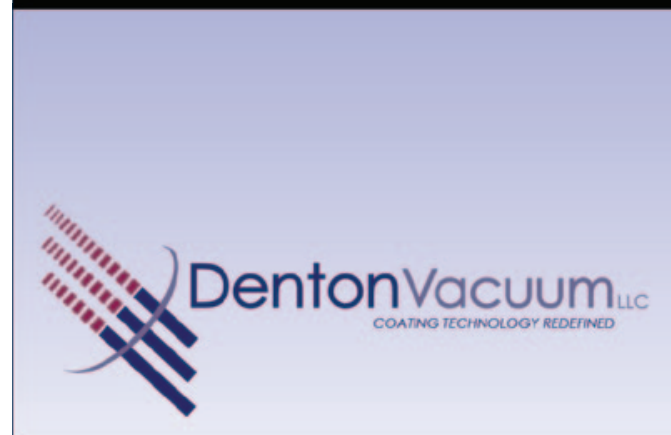

Call us today at 800-666-6004

or visit us online at www.dentonvacuum.com 


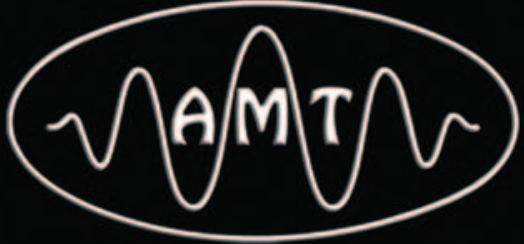

High Definition Digital

TEM Cameras with

1 to 16 Megapixels
- AMT SOLUTIONS

- Life Science Cameras

- Material Science Cameras

- Easy To Use Software

- Reliability and Services

- TEM Integration

- Extensive Support
